\title{
A lung ultrasound score for early triage of elderly patients with acute dyspnea
}

\author{
Thibaut Markarian, MD*; Laurent Zieleskiewicz, MD+; Gilles Perrin, MD*; Pierre-Géraud Claret, MD, PhD; \\ Anderson Loundou, $\mathrm{MD}^{\S}$; Pierre Michelet, $\mathrm{MD}, \mathrm{PhD}^{*}$; Xavier Bobbia, $\mathrm{MD}^{*} \ddagger$
}

\section{CLINICIAN'S CAPSULE}

What is known about the topic?

A lung ultrasound has good accuracy in finding the cause of acute dyspnea.

What did this study ask?

Can a simple lung ultrasound score, done early, detect dyspneic patients who will require intensive care?

What did this study find?

A modified lung ultrasound (MLUS) has very good accuracy in predicting intensive care unit admission and/or death.

Why does this study matter to clinicians?

Dyspneic patients with a high MLUS should be monitored and treated early.

\section{ABSTRACT}

Objectives: Lung ultrasound has value in diagnosing dyspnea. The main objective of this study was to evaluate the accuracy of a modified lung ultrasound (MLUS) score to predict the severity of acute dyspnea in elderly patients.

Methods: This was an observational single-centre study including patients over age 64 admitted to the emergency department for acute dyspnea with hypoxia. Participants had an early lung ultrasound performed by a dedicated emergency physician, followed by the usual care by a team blinded to the lung ultrasound results. Patients were allocated by disposition to either a critical care (CC) group (patients who needed admission to the intensive care unit [ICU] and/or who died within 48 h) or a standard care group.

Results: Among 137 patients analysed (mean age $79 \pm 13$ years, 74 [54\%] women), 43 (31\%) were categorized into the CC group. The time taken to obtain the MLUS was $30 \pm$ $22 \mathrm{~min}$. The area under the receiver operating characteristic curve of the MLUS for predicting the CC group was 0.97
$(0.92-0.99 ; p<0.01)$ with a cut-off set strictly above 17 for $93 \%$ sensitivity (81-99), 99\% specificity (94-100), a positive predictive value of $98 \%(87-100)$, a negative predictive value of $97 \%$ (91-99), a positive likelihood ratio of 86 , a negative likelihood ratio of 0.07 , and a diagnostic accuracy of $97 \%$ (93-99). In a multivariate analysis, the MLUS was the only independent associated factor for the CC group.

Conclusion: An early lung ultrasound score can predict the need for ICU admission and/or death within 48 hours in elderly dyspneic patients.

\section{RÉSUMÉ}

Objectif: L'échographie pulmonaire (EP) a une valeur diagnostique dans la dyspnée. L'étude avait pour objectif principal d'évaluer l'exactitude du score d'échographie pulmonaire modifiée (EPM) aux fins de prévision du degré de gravité de la dyspnée aiguë chez les personnes âgées.

Méthode: Il s'agit d'une étude d'observation monocentrique, menée chez des patients âgés de plus de 64 ans et admis au service des urgences pour de la dyspnée aiguë, accompagnée d'hypoxie. Les participants ont été soumis à une EP précoce, effectuée par un urgentologue spécialisé en la matière, puis ont reçu les soins usuels donnés par des professionnels tenus dans l'ignorance des résultats de l'EP. Les patients ont été classés, selon les suites à donner, dans le groupe de soins intensifs (SI) (patients admis au service de soins intensifs [SSI] ou morts au cours des $48 \mathrm{~h}$ suivant leur arrivée) ou dans le groupe de soins usuels (SU).

Résultats: Les dossiers de 137 patients ont été analysés (âge moyen : $79 \pm 13$ ans; femmes : $74[54 \%]$ ) et, sur ce nombre, $43(31 \%)$ ont été classés dans le groupe de SI. Le temps d'attente des résultats de l'EPM s'est établi à $30 \pm 22 \mathrm{~min}$. La valeur prévisionnelle de la surface sous la courbe de I'EPM pour le classement des patients dans le groupe de SI était de 0,97 $(0,92-0,99 ; p<0,01)$, et la valeur-seuil a été établie rigoureusement au-dessus de 17, d'où une sensibilité de 93\% (81-99); une

From the *Department of Emergency Medicine and Intensive Care, Timone University Hospital, Marseille, France; †Department of Anesthesiology and Intensive Care, North Hospital, APHM, Aix-Marseille University, Marseille, France; ‡Department of Anesthesiology, Emergency Medicine and Critical Care, Intensive Care Unit, Nîmes University Hospital, Nîmes, France; and the §Department of Public Health, EA3279 Self-Perceived Health Assessment Research Unit, Timone University, Aix-Marseille University, Marseille, France.

Correspondence to: Dr. Xavier Bobbia, Pôle ARDU CHU Nîmes, Place du Pr Robert Debré 30029, Nîmes, France; Email: xavier.bobbia@ gmail.com 
spécificité de 99\% (94-100); une valeur prévisionnelle positive de 98\% (87-100); une valeur prévisionnelle négative de $97 \%$ (91-99); un rapport de vraisemblance positif de 86; un rapport de vraisemblance négatif de 0,07 et une exactitude diagnostique de 97\% (93-99). D'après l'analyse plurifactorielle, le score d'EPM était le seul facteur indépendant associé au groupe de SI.
Conclusion: Le score d'EP précoce offre une valeur prévisionnelle quant à la nécessité $d^{\prime}$ admettre des personnes âgées dyspnéiques au SSI ou à la mort dans les $48 \mathrm{~h}$ suivant leur arrivée.

Keywords: dyspnea, emergency medicine, lung, point-of-care systems, triage, ultrasonography

\section{INTRODUCTION}

Dyspnea, a frequently encountered presentation in the emergency department (ED), ${ }^{1,2}$ can manifest with many different degrees of clinical severity. ${ }^{3}$ Although dyspnea is a subjective symptom, it represents a severe symptom for many conditions and has been associated with significant morbidity and mortality, ${ }^{4,5}$ notably in cardiovascular $^{6}$ and chronic respiratory diseases. ${ }^{7}$ Although there are degrees of severity of disease scores, ${ }^{8,9}$ they are only recorded in intensive care units (ICUs), and their low sensitivity limits their use in triage assessment on an individual basis. ${ }^{10,11}$ Dyspnea is also problematic when it comes to diagnosis, ${ }^{2}$ especially for elderly patients where elements of their medical history, such as cardiovascular and respiratory symptoms, are intricately linked. ${ }^{3,12}$ Lung ultrasound has value in diagnosing dyspnea. ${ }^{13-17}$ Specifically, it allows us to assess lung aeration and the location and severity of pulmonary edema. Point-of-care ultrasound (POCUS) can quickly help stratify dyspneic ED patients who should undergo a more detailed evaluation. ${ }^{18}$ For ICU patients, lung ultrasound has reportedly played a role in determining the diagnosis, prognosis, and clinical severity, ${ }^{19-24}$ but the value of an early evaluation by lung ultrasound for the triage of dyspneic ED patients has not been known.

The objective of this study was to evaluate the accuracy of early lung ultrasound scores in predicting the severity of acute dyspnea in patients over age 64 .

\section{METHODS}

\section{Materials}

This observational single-centre study was conducted from November 2015 to March 2016. The study protocol was approved by the ethics committee of the French Society of Anesthesia and Intensive Care Medicine, who waived the requirement for informed consent because the study was observational. We declared our study to the National Commission for Data Processing and Civil Liberties (CNIL). The study analysed the records of patients admitted for acute dyspnea in an ED of the University Hospital of Marseille. Approximately 83,000 patients a year are admitted to this ED.

\section{Patients}

Because an association between diagnosis and prognosis is shown in this population, ${ }^{3}$ we chose to study 64-year-old dyspneic patients. Patients were included if they were over age 64, were admitted for acute dyspnea, were on spontaneous ventilation, and presented with oxygen saturation levels measured by pulse oximeter $\left(\mathrm{SpO}_{2}\right)$ that were strictly below $94 \%$ in room air. Patients with a history of lung surgery or pneumothorax were not included, due to the inapplicability of the ultrasound score.

\section{Aim}

The objective of our study was to determinate the accuracy of a lung ultrasound score (modified lung ultrasound [MLUS] score) obtained upon admission of acutely dyspneic patients over 64 years of age to predict ICU admission or death within 48 hours of ED admission. The primary end point was either admission to an ICU after the ED or patient death within 48 hours of ED admission. Patients meeting these criteria were included in the critical care (CC) group. It was the intensivist who made the decision to admit the patient to the ICU. If the intensivist thought that the patient was serious enough to be admitted to the ICU, although an end-of-life decision was decided upon, then the patient was classified in this group. The notion of ICU nonadmission according to an end-of-life decision was sought in the medical files. 
Patients without these criteria were included in the standard care (SC) group.

\section{Measurements}

Epidemiological data collected included gender, age, vital signs on hospital admission, medical history of cardiovascular or respiratory problems, and cardiovascular risk factors as well as the dyspnea score according to the New York Heart Association (NYHA) classification system. ${ }^{25}$ The final diagnosis was based on the electronic medical record. The time to obtain the MLUS score was considered the time between ED admission and the obtaining of the score.

\section{Procedure}

All patients meeting the inclusion criteria received a lung ultrasound and a calculation of an MLUS score upon admission to the ED. Five emergency physicians (EPs) performed the ultrasound. All EPs were certified with a university diploma in clinical ultrasound and routinely performed a lung ultrasound. One EP in particular was present to do the lung ultrasound for the study. The operators did not perform a clinical exam on the patients. The patients then received SC from a team blinded to the MLUS results. The responsible care physician did not know the results of the MLUS and could do a POCUS.

\section{Ultrasound (Figure 1)}

The study used a portable Logiq E ultrasound system manufactured by General Electric (GE Healthcare, Milwaukee, Wisconsin, USA) with a $1-5 \mathrm{MHz}$ sector array transducer, only using the 2D mode. A lung ultrasound was carried out to calculate the MLUS score. Here, this score will be called the MLUS (the modified LUS [lung ultrasound (score)]) as described by Bouhemad et al. ${ }^{26}$ Both lungs were examined with the patient in a supine or semi-seated position, covering six zones on each lung. Thus, there were a total of 12 zones, 2 anterior, 2 lateral, and 2 posterior zones per lung, as described by Bouhemad et al. ${ }^{26}$ (see Figure 1). Four types of lung aeration were evaluated according to international guidelines $^{27}$ (see Figure 1), as follows:
- Normal aeration (0 points), corresponding to the presence of lung sliding associated with A-lines or fewer than three B-lines

- Moderate damage (1 point), suggesting interstitial syndrome, with B-lines of $7 \mathrm{~mm}$ apart or less, that is, at least three B-lines on the same ultrasound

- Serious damage (2 points) corresponding to alveolar interstitial syndrome, with B-lines of $3 \mathrm{~mm}$ apart or less

- Pulmonary consolidation and/or pleural effusion, with a minimum distance of $20 \mathrm{~mm}$ or more between the parietal and visceral pleurae on a transverse scan (3 points).

We thought we should highlight and evaluate potential pleural effusion, which could affect the short-term prognosis. ${ }^{27}$ In cases where the quality of the examination was considered poor (i.e., uninterpretable), the patient was excluded from the study.

\section{Number of subjects}

The sample size was calculated based on a study by Ray et al., ${ }^{3}$ who found that the rate of ICU admission was $29 \%$. To have a specificity of $95 \%$ with a two-tailed alpha risk of 0.05 , we needed to include 104 subjects, with 30 patients requiring ICU admission. To allow for any data loss, we planned to include 5\% more patients. Given the number of dyspneic patients usually admitted in our ED during the period, records were taken during eight random periods of 5 days each, spread over the 5 months of the study.

\section{Data analysis}

Quantitative data are expressed as mean \pm standard deviation (SD) or median with the 25 th and 75 th percentiles (25th percentile-75th percentile), according to the variable distribution. The qualitative variables are expressed as the frequency with percentage. The variables compared between the two groups included age, gender, past medical history (cardiac history, diabetes, hypertension, dyslipidemia, current smoker, lung history), clinical state (oxygen saturation, respiratory rate, dyspnea NYHA class, systolic arterial pressure, temperature, Glasgow coma score), the Boston score, ${ }^{27}$ the Geneva score, the serum lactate level, and the MLUS. A comparison of quantitative variables among the different 


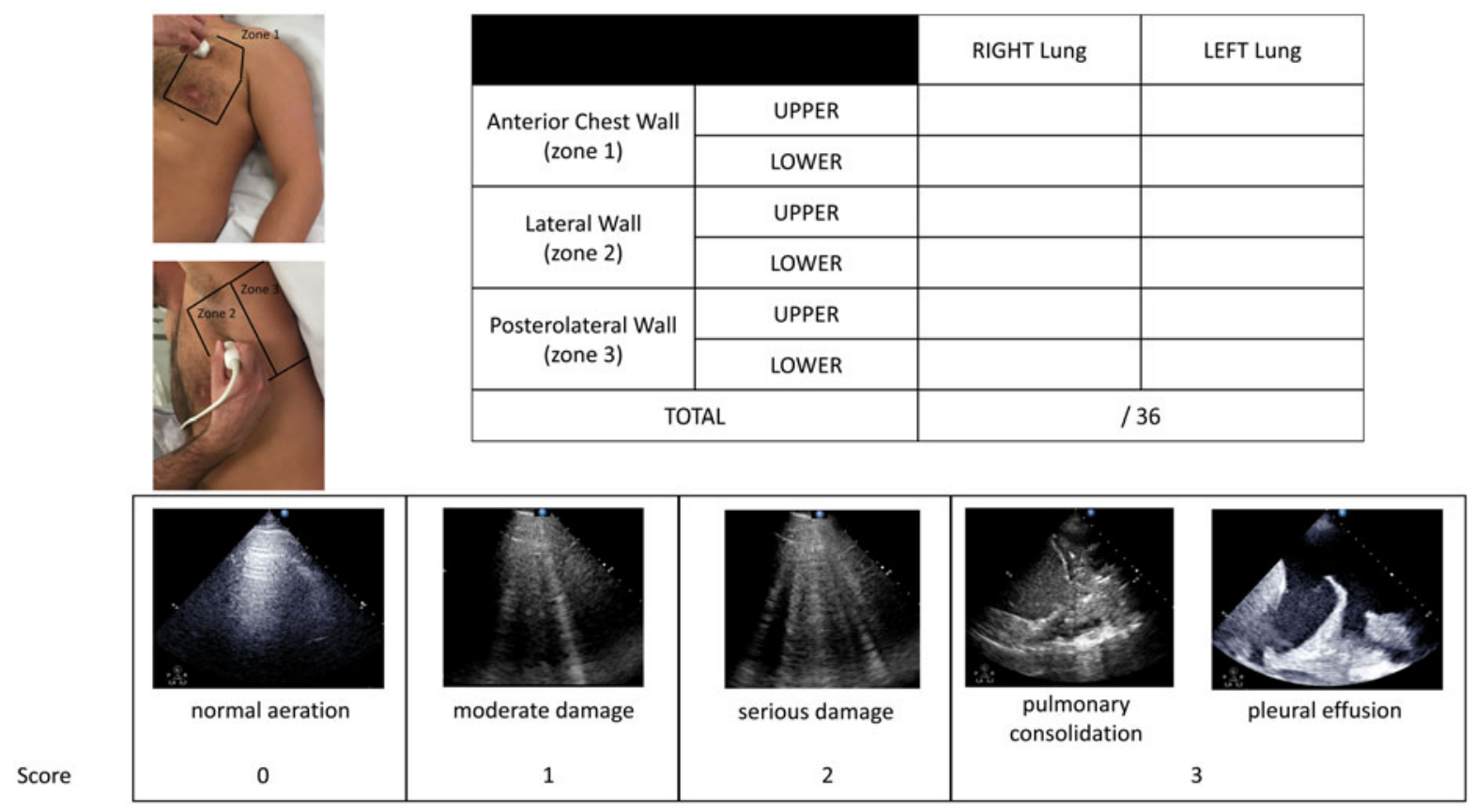

Figure 1. Modified lung ultrasound score calculation.

groups was performed via the student's $t$-test. When the conditions of validity of this test were not verified, the nonparametric Mann-Whitney U test was used. The chi-square or Fisher exact test was used for qualitative variables. We also performed a multivariate analysis using a multiple logistic regression. Variables with a significance level of at least 0.1 were included in the logistic model. The receiver operating characteristic (ROC) curve analysis was used to assess the discriminatory power of the prognostic factor. Subsequently, the optimal cut-off points were determined via the ROC curve based on the Youden index. All tests were two-tailed. The statistical significance was defined as $p<0.05$. Statistical analysis was performed using PASW Statistics version 20 (IBM SPSS Inc., Chicago, IL, USA).

\section{RESULTS}

\section{Participants}

During the data collection period, 520 patients over age 64 years were admitted to the ED. Among these patients, $140(27 \%)$ received an early lung ultrasound. Within this population, $3(2 \%)$ were excluded from the study due to a history of pneumonectomy. There were no exclusions reported for mediocre ultrasound imaging. The five different operators performed a number of ultrasound scans as follows: 18 (13\%), 21 (15\%), 26 (19\%), 32 (24\%), and 40 (29\%). Epidemiological data are provided in Table 1.

\section{Outcomes}

Among the patients who had the protocol analysis, 94 $(69 \%)$ were categorized into the SC group, whereas 43 (31\%) were put into the CC group. In the CC group, $14(33 \%)$ patients died while staying in the hospital. Epidemiological data for both populations are provided in Table 1 . The MLUS was calculated $30 \pm 22$ minutes after a patient arrived in the ED. The area under the ROC curve for predicting admission to the ICU or death within 48 hours, using MLUS, was 0.97 (0.92$0.99 ; p<0.01)$, with a cut-off set strictly above 17 for 93\% sensitivity (81-99), 99\% specificity (94-100), a positive predictive value of $98 \%$ (87-100), a negative predictive value of 97\% (91-99), a positive likelihood ratio of 86 , a negative likelihood ratio of 0.07 , and a diagnostic accuracy of 97\% (93-99) (Figure 2). Univariate analysis of the differences between the CC and SC groups revealed factors linked to clinical presentation, lactate concentration, and the MLUS. For the MLUS, a cut-off set at 17 seemed particularly discriminating. In the multivariate analysis, the MLUS was the only 


\begin{tabular}{|c|c|c|c|c|}
\hline Characteristics & $\begin{array}{c}\text { All patients } \\
n=137\end{array}$ & $\begin{array}{l}\text { Standard care group } \\
\qquad n=94\end{array}$ & $\begin{array}{l}\text { Critical care group } \\
\qquad n=43\end{array}$ & $p$-value \\
\hline Age (years) & $79 \pm 13$ & $78 \pm 14$ & $81 \pm 11$ & 0.32 \\
\hline Female & $74(54 \%)$ & $52(55 \%)$ & $22(51 \%)$ & 0.65 \\
\hline Cardiac history & $76(55 \%)$ & $48(51 \%)$ & $28(65 \%)$ & 0.13 \\
\hline Diabetes mellitus & $34(25 \%)$ & $24(26 \%)$ & $10(23 \%)$ & 0.78 \\
\hline HTA & $78(57 \%)$ & $54(57 \%)$ & $24(56 \%)$ & 0.86 \\
\hline Dyslipidemia & $25(18 \%)$ & $12(13 \%)$ & $13(30 \%)$ & 0.01 \\
\hline Current smokers & $26(19 \%)$ & $20(21 \%)$ & $6(14 \%)$ & 0.31 \\
\hline Lung history & $56(41 \%)$ & $35(37 \%)$ & $21(49 \%)$ & 0.20 \\
\hline $\mathrm{SpO}_{2}$ prior to oxygen (\%) & $91 \pm 7$ & $92 \pm 7$ & $87 \pm 7$ & $<0.01$ \\
\hline Respiratory rate (n/minutes) & $24 \pm 8$ & $22 \pm 6$ & $29 \pm 8$ & $<0.01$ \\
\hline Dyspnea, NYHA class & & & & $<0.01$ \\
\hline 1 & $6(4 \%)$ & $6(7 \%)$ & $0(0 \%)$ & \\
\hline 2 & $9(7 \%)$ & $9(10 \%)$ & $0(0 \%)$ & \\
\hline 3 & $20(15 \%)$ & $16(17 \%)$ & $4(9 \%)$ & \\
\hline 4 & $100(73 \%)$ & $61(66 \%)$ & $39(91 \%)$ & \\
\hline Systolic arterial pressure (mmHg) & $138 \pm 28$ & $138 \pm 27$ & $139 \pm 30$ & 0.89 \\
\hline Temperature $\left({ }^{\circ} \mathrm{C}\right)$ & $36.9 \pm 1$ & $37 \pm 1$ & $36.8 \pm 0.8$ & 0.44 \\
\hline Glasgow coma score & $15[15 ; 15]$ & $15 \pm 1$ & $15 \pm 1$ & 0.32 \\
\hline Boston score & $8 \pm 2$ & $7 \pm 2$ & $9 \pm 2$ & $<0.01$ \\
\hline Geneva score & $4 \pm 3$ & $4 \pm 3$ & $5 \pm 3$ & 0.41 \\
\hline MLUS score & $9[3 ; 9]$ & $5[1 ; 9]$ & $23[20 ; 26]$ & $<0.01$ \\
\hline Lactate (mmol/L) & $2.0[1.0 ; 1.9]$ & $1.4[0.9 ; 1.7]$ & $1.8[1.3 ; 3.9]$ & 0.01 \\
\hline Final diagnoses & & & & $<0.01$ \\
\hline $\mathrm{CPO}$ & $37(27 \%)$ & $11(12 \%)$ & $26(60 \%)$ & \\
\hline Pneumonia & $42(31 \%)$ & $36(38 \%)$ & $6(14 \%)$ & \\
\hline Asthma/COPD & $10(7 \%)$ & $9(10 \%)$ & $1(3 \%)$ & \\
\hline Combined causes & $20(15 \%)$ & $13(14 \%)$ & $7(16 \%)$ & \\
\hline Other & $28(20 \%)$ & $25(27 \%)$ & $3(7 \%)$ & \\
\hline
\end{tabular}

remaining prognostic factor independent of severity with a cut-off at 17 (Table 2).

\section{DISCUSSION}

\section{Main results}

An LUS recorded on admission to the ED efficiently allowed us to triage dyspneic patients over 64 years old. In other words, its calculation was associated with the rapid and adaptive triage of elderly patients with dyspnea. In the context of an ED, it is important to determine what constitutes a reasonable amount of time for conducting this evaluation. Zanobetti et al. showed a good concordance between the diagnoses reached after POCUS evaluation and after standard ED evaluation in the differential diagnosis of dyspneic patients, with a POCUS diagnosis time of 24 minutes. ${ }^{18}$ The authors concluded that POCUS may help stratify patients who should undergo a second-level diagnostic test. Our study showed that, in approximately the same time, the calculation of the MLUS score accurately predicted the outcome for severely ill patients.

\section{Originality of the study}

Dyspnea management in the ED includes both rapid hypothetical diagnosis and patient-oriented triage. The value of lung ultrasound in diagnosing dyspnea has 


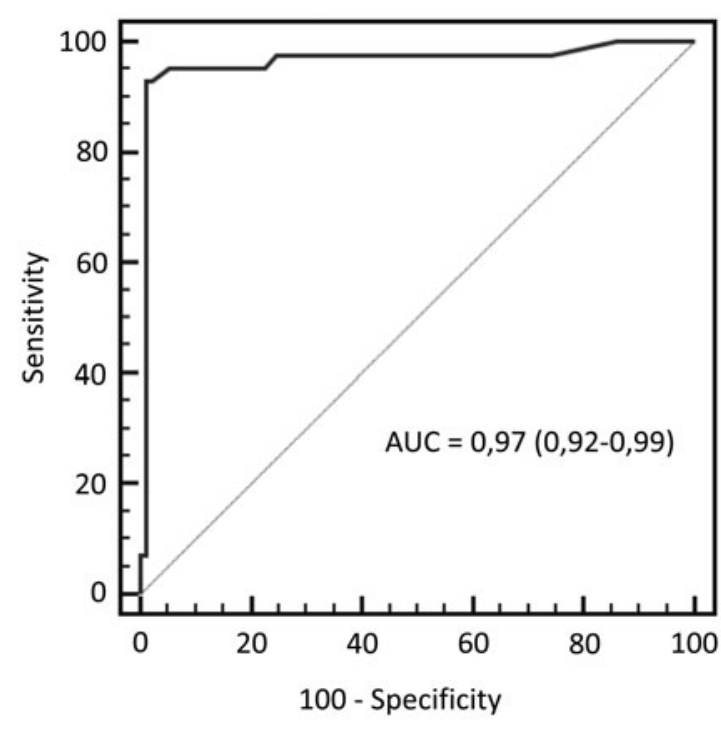

Figure 2. Receiver operating characteristic curve depicting the relationship between the modified lung ultrasound score with a cut-off set strictly above 17 and the critical care group.

\begin{tabular}{|c|c|c|}
\hline & \multicolumn{2}{|c|}{ Multivariate analysis } \\
\hline & OR $(95 \% \mathrm{Cl})$ & $p$-value \\
\hline Dyslipidemia & $0.1(0-21.5)$ & 0.38 \\
\hline Respiratory rate & $1.3(0.9-2.0)$ & 0.21 \\
\hline Boston score & $1.3(0.6-3.1)$ & 0.52 \\
\hline MLUS score > 17 & 454.9 (9.9-20984) & $<0.01$ \\
\hline Lactate & $1.7(0.5-6.0)$ & 0.43 \\
\hline
\end{tabular}

already been proven. ${ }^{13-18,27-31}$ Lung ultrasound is more accurate than chest X-rays to diagnose the main causes of acute dyspnea. ${ }^{30-35}$ The value of lung ultrasound in prognosis has also been indicated for patients suffering from dyspnea or chest pain ${ }^{36}$ and acute respiratory distress syndrome..$^{22,23,37}$ If the diaphragmatic excursion measurement can predict the use of a ventilation technique, ${ }^{38}$ then the value of lung ultrasound in helping with patient triage on admission to the ED has not yet been reported clearly. The additional diagnostic value of simultaneous echocardiogram and lung ultrasound recordings is also obvious, but it is time-consuming and, therefore, is less applicable in the context of triage. ${ }^{30}$ We chose to change an existing score: the LUS. ${ }^{26}$ This score seemed simple to achieve and was described in critical patients.

\section{Limits}

Nevertheless, our study has its limits. Firstly, this study is monocentric; therefore, multicentre studies will probably be required to confirm our results and to assess the MLUS score as a prognostic score for dyspnea. Secondly, none of the patients were followed up more than 48 hours post-admission, and only initial data were taken into account when categorizing patients into the CC and SC groups. It would be advisable to study the population's progress over at least 30 days to more accurately evaluate the usefulness of an early LUS as a prognostic factor. Thirdly, if the operators did not perform a clinical exam on the patients, then the operators saw the patients. It is possible that the clinical state of the patients changed after their ultrasound interpretation. Fourthly, there is little evidence for the diagnostic interest of pulmonary ultrasound scoring. Finally, in our study, most ICU patients had cardiogenic pulmonary edema (CPO), and the majority of CPO patients went to the ICU. It is possible that the accuracy of the MLUS would have been lower in a different distribution of diagnoses.

\section{CONCLUSION}

Our study showed that an early LUS can predict with good accuracy the clinical severity of elderly dyspneic patients, as defined by the need for ICU admission and/or death within 48 hours of being admitted to the ED.

Competing interests: $\mathrm{XB}$ and $\mathrm{LZ}$ declare a competing interest as a U.S. teacher for GE (GE Medical Systems Ultrasound) customers. The other authors state that they have no competing interests.

\section{REFERENCES}

1. Malas O, Cağlayan B, Fidan A, et al. Cardiac or pulmonary dyspnea in patients admitted to the emergency department. Respir Med 2003;97(12):1277-81.

2. Mueller C, Scholer A, Laule-Kilian K, et al. Use of B-type natriuretic peptide in the evaluation and management of acute dyspnea. N Engl 7 Med 2004;350(7):647-54.

3. Ray P, Birolleau S, Lefort $Y$, et al. Acute respiratory failure in the elderly: etiology, emergency diagnosis and prognosis. Crit Care Lond Engl 2006;10(3):R82.

4. Vinson JM, Rich MW, Sperry JC, et al. Early readmission of elderly patients with congestive heart failure. 7 Am Geriatr Soc 1990;38(12):1290-5.

5. Marrie TJ. Community-acquired pneumonia in the elderly. Clin Infect Dis 2000;31(4):1066-78. 
6. Abidov A, Rozanski A, Hachamovitch R, et al. Prognostic significance of dyspnea in patients referred for cardiac stress testing. N Engl f Med 2005;353(18):1889-98.

7. Celli BR, Cote CG, Marin JM, et al. The body-mass index, airflow obstruction, dyspnea, and exercise capacity index in chronic obstructive pulmonary disease. $N$ Engl $7 \mathrm{Med}$ 2004;350(10):1005-12.

8. Le Gall JR, Loirat P, Alperovitch A. Simplified acute physiological score for intensive care patients. Lancet Lond Engl 1983;2(8352):741.

9. Knaus WA, Draper EA, Wagner DP, Zimmerman JE. APACHE II: a severity of disease classification system. Crit Care Med 1985;13(10):818-29.

10. Lemeshow S, Klar J, Teres D. Outcome prediction for individual intensive care patients: useful, misused, or abused? Intensive Care Med 1995;21(9):770-6.

11. Glance LG, Osler T, Shinozaki T. Intensive care unit prognostic scoring systems to predict death: a cost-effectiveness analysis. Crit Care Med 1998;26(11):1842-9.

12. Lien CTC, Gillespie ND, Struthers AD, McMurdo MET. Heart failure in frail elderly patients: diagnostic difficulties, co-morbidities, polypharmacy and treatment dilemmas. Eur 7 Heart Fail 2002;4(1):91-8.

13. Lichtenstein $D$, Mezière $G$. A lung ultrasound sign allowing bedside distinction between pulmonary edema and COPD: the comet-tail artifact. Intensive Care Med 1998;24(12): 1331-4.

14. Lichtenstein DA, Mezière GA. Relevance of lung ultrasound in the diagnosis of acute respiratory failure: the BLUE protocol. Chest 2008;134(1):117-25.

15. Liu Z-P, Zhang Y, Bian H, et al. Clinical application of rapid B-line score with lung ultrasonography in differentiating between pulmonary infection and pulmonary infection with acute left ventricular heart failure. Am 7 Emerg Med 2016;34(2):278-81.

16. Xirouchaki N, Kondili E, Prinianakis G, et al. Impact of lung ultrasound on clinical decision making in critically ill patients. Intensive Care Med 2014;40(1):57-65.

17. Volpicelli G, Mussa A, Garofalo G, et al. Bedside lung ultrasound in the assessment of alveolar-interstitial syndrome. $\mathrm{Am}$ 7 Emerg Med 2006;24(6):689-96.

18. Zanobetti M, Scorpiniti M, Gigli C, et al. Point-of-care ultrasonography for evaluation of acute dyspnea in the ED. Chest 2017;151(6):1295-301.

19. Bouhemad B, Zhang M, Lu Q, Rouby J-J. Clinical review: bedside lung ultrasound in critical care practice. Crit Care Lond Engl 2007;11(1):205.

20. Bouhemad B, Liu Z-H, Arbelot C, et al. Ultrasound assessment of antibiotic-induced pulmonary reaeration in ventilatorassociated pneumonia. Crit Care Med 2010;38(1):84-92.

21. Soummer A, Perbet S, Brisson H, et al. Ultrasound assessment of lung aeration loss during a successful weaning trial predicts postextubation distress. Crit Care Med 2012;40(7):2064-72.

22. Li L, Yang Q, Li L, et al. [The value of lung ultrasound score on evaluating clinical severity and prognosis in patients with acute respiratory distress syndrome]. Zhonghua Wei Zhong Bing Fi fiu Yi Xue 2015;27(7):579-84.
23. Zhao Z, Jiang L, Xi X, et al. Prognostic value of extravascular lung water assessed with lung ultrasound score by chest sonography in patients with acute respiratory distress syndrome. BMC Pulm Med 2015;15:98.

24. Mongodi S, Via G, Girard M, et al. Lung ultrasound for early diagnosis of ventilator-associated pneumonia. Chest 2016;149 (4):969-80.

25. Hurst JW, Morris DC, Alexander RW. The use of the New York Heart Association's classification of cardiovascular disease as part of the patient's complete problem list. Clin Cardiol 1999;22(6):385-90.

26. Bouhemad B, Mongodi S, Via G, Rouquette I. Ultrasound for "lung monitoring" of ventilated patients. Anesthesiology 2015;122(2):437-47.

27. Volpicelli G, Elbarbary M, Blaivas M, et al. International evidence-based recommendations for point-of-care lung ultrasound. Intensive Care Med 2012;38(4):577-91.

28. Pivetta E, Goffi A, Lupia E, et al. Lung ultrasound-implemented diagnosis of acute decompensated heart failure in the ED: a SIMEU multicenter study. Chest 2015;148(1):202-10.

29. Silva S, Biendel C, Ruiz J, et al. Usefulness of cardiothoracic chest ultrasound in the management of acute respiratory failure in critical care practice. Chest 2013;144(3):859-65.

30. Bataille B, Riu B, Ferre F, et al. Integrated use of bedside lung ultrasound and echocardiography in acute respiratory failure: a prospective observational study in ICU. Chest 2014;146 (6):1586-93.

31. Laursen CB, Sloth E, Lassen AT, et al. Point-of-care ultrasonography in patients admitted with respiratory symptoms: a single-blind, randomised controlled trial. Lancet Respir Med 2014;2(8):638-46.

32. Xirouchaki N, Magkanas E, Vaporidi K, et al. Lung ultrasound in critically ill patients: comparison with bedside chest radiography. Intensive Care Med 2011;37(9):1488-93.

33. Inglis AJ, Nalos M, Sue K-H, et al. Bedside lung ultrasound, mobile radiography and physical examination: a comparative analysis of diagnostic tools in the critically ill. Crit Care Resusc 7 Australas Acad Crit Care Med 2016;18(2):124.

34. Blaivas M, Lyon M, Duggal S. A prospective comparison of supine chest radiography and bedside ultrasound for the diagnosis of traumatic pneumothorax. Acad Emerg Med Off 7 Soc Acad Emerg Med 2005;12(9):844-9.

35. Lichtenstein D, Goldstein I, Mourgeon E, et al. Comparative diagnostic performances of auscultation, chest radiography, and lung ultrasonography in acute respiratory distress syndrome. Anesthesiology 2004;100(1):9-15.

36. Frassi F, Gargani L, Tesorio P, et al. Prognostic value of extravascular lung water assessed with ultrasound lung comets by chest sonography in patients with dyspnea and/ or chest pain. 7 Card Fail 2007;13(10):830-5.

37. Leblanc D, Bouvet C, Degiovanni F, et al. Early lung ultrasonography predicts the occurrence of acute respiratory distress syndrome in blunt trauma patients. Intensive Care Med 2014;40(10):1468-74.

38. Bobbia X, Clément A, Claret PG, et al. Diaphragmatic excursion measurement in emergency patients with acute dyspnea: toward a new diagnostic tool? Am f Emerg Med 2016;34(8):1653-7. 